\title{
Research status of semi-permanent release agent
}

\author{
Bo Liang ${ }^{1,2, *}$, Zhonghuan Zhang ${ }^{2}$, Tong Yin ${ }^{2}$, Shuai Yang ${ }^{2}$, Junpeng Shao ${ }^{2}$, William Manchester ${ }^{3}$ \\ ${ }^{1}$ Shandong Meichen Industrial Group Co., LTD., Zhucheng 262200, China \\ ${ }^{2}$ Harbin University of Science and Technology, Harbin 150000, China \\ ${ }^{3}$ Krirk University, Bangkok 10220, Thailand
}

\begin{abstract}
According to the composition of substances, release agent can be divided into organic and inorganic two types, inorganic release agent includes mica powder, graphite powder, etc., organic release agent mainly includes organic silicon, organic fluorin. According to the service life, release agent can be divided into onetime release agent and semi-permanent release agent. This paper introduced several kinds of releasing agent, summarized and analyzed the advantages of semi-permanent release agent and the present situation of domestic and foreign research, and prospected the future of release agent.
\end{abstract}

Keywords: Release agent, Semi-permanent release agent, Rubber products

\section{Introduction}

China is a major producer and consumer of plastic and rubber industry. Since 2010, the annual production of plastic and rubber has exceeded 60 million tons, ranking first in the world in both annual production and consumption capacity. With the rise of rubber plastic industry, the amount of release agent is also increasing year by year, so the market size is huge[1-9].

Especially in the automobile hose industry, the molding of automobile hose needs to go through plasticizing, mixing, pressing, calendering and vulcanization several processes. Vulcanization is an important process to make rubber and skeleton layer produce high adhesive strength, so that it becomes a whole, is an important link to ensure the quality of rubber hose. In the process of vulcanization, the rubber hose and the mandrel are easy to adhesion, so before vulcanization, the mandrel needs to be coated with a release agent to prevent adhesion[10-15]. However, there are many problems such as oil bleaching, yellowing, poor stability and less demoulding times in the current rubber hose demoulding agent [1, 16-22].

Due to the effect of high temperature for a long time, the product will form dirt on the mold in the casting process, resulting in the phenomenon of product adhesion, so it is necessary to form a durable protective film on the product to ensure the smooth demoulding. The silicone release agent is an important additive to form this layer of protective film. The organosilicon compound has a variety of practical functions, which has a short film forming time at low temperature, good high temperature resistance, is often used in the demoulding process of a basic raw material[23-25].

Because of the existence of silicon-oxygen bond in its molecular structure, the bond energy and bond angle of organosilicon compound in the organosilicon demoulding agent are larger. Therefore, it has high and low temperature resistance, radiation resistance, oxidation resistance, low surface tension, hydrophobicity, electrical insulation and other characteristics. Because of these properties, organosilicon compounds are widely used in textile industry, food industry and construction industry [26-29].

However, with the improvement of people's living standard and the increase of industry demand, the functional requirements of organic silicon are also gradually increasing[30-36]. At the same time, due to the large hydrophobicity of methyl silicone oil, it is difficult to emulsify and the microemulsion stability is not good, resulting in oil and water separation, oil bleaching, yellowing and other problems in the process of use, so that the demoulding effect becomes poor. In order to meet the requirements of social industry progress, and improve the technical problems of methyl silicone oil release agent instability, it is necessary to reform the structure of organic silicon compound, to obtain more excellent and complete performance.

The rubber products industry in the world is booming and the rubber output is rising steadily. As a big country of rubber production and consumption, the rubber industry has been in a state of rapid development since the $21 \mathrm{st}$ century. With the rise of rubber industry, release agent as an indispensable additive rubber products have also been developed, a large number of domestic and foreign research on release agent has been launched. In the process of vulcanization of rubber products, when the products are peeled from the mold, friction resistance may be generated because of the surface defect of the mold,

\footnotetext{
* Corresponding author: bliang0325@163.com
} 
which increases the difficulty of peeling[37]. Release agent can not only reduce the product and mold peeling force to decrease product demoulding difficulty, but also protect the product and mold surface. It plays an irreplaceable role in the vulcanization of rubber products.

\section{Classification of release agent}

According to the composition of substances, release agent can be divided into organic and inorganic two types, inorganic release agent includes mica powder, graphite powder, etc., organic release agent mainly includes organic silicon, organic fluorine, wax system, etc[38]. Silicone series and fluorine series are widely used because of their excellent physical and chemical properties.

According to the service life, release agent can be divided into one-time release agent and semi-permanent release agent $[37,39]$. The release agent is coated on the surface of the mold before each production cycle, and demoulding is achieved by destroying the cohesiveness of the release agent, which is called the disposable release agent. The main disadvantage of disposable release agent is that when the product is released from the mold, most of the release agent will migrate to the surface of the product resulting in huge loss and decrease in the accuracy of the product, so the disposable release agent is also known as migration release agent or sacrifice release agent.

Semi-permanent release agent is generally made of fluorine and silicon crosslinked polymer as the main raw material. The semi-permanent release agent is applied to the surface of the mold. The solvent volatilizes at room temperature or high temperature, and the solute is crosslinked and solidified, forming a complete film on the surface of the mold. Compared with the disposable release agent, the formation of the cross-linked film greatly improves the release performance. Due to the large adhesion of the cross-linked film, the release agent does not migrate to the product after the product molding demoulding process. so the semi-permanent release agent is also known as the non-migratory release agent. Due to the little consumption of release agent in the production process, it not only reduces the raw material cost but also reduces the manual cleaning mold time, greatly improving the production efficiency. so semi-permanent release agent is widely used, semi-permanent release agent accounts for up to $25 \%$ in the rubber industry[37, 40-44].

\section{Superiority of semi-permanent release agent}

Compared with other types of release agent, semipermanent release agent will form a chemically stable and heat-resistant crosslinking film on the surface of the mold, because this crosslinking film is difficult to be decomposed, so semi-permanent release agent has the following advantages[40, 42, 44, 45]:

(1) In the demoulding process of vulcanized rubber products, the pollution of the mold caused by the migration of paraffin series release agent is avoided.

(2) The friction coefficient of crosslinked film is very small, which can protect rubber products and molds well and ensure that the surface of molds remains smooth

(3) There is no need to worry about subsequent bond failures.

(4) Because of the stable chemical properties of the crosslinked membrane, there is no need to worry about its reaction with the decomposition of rubber in the high temperature of vulcanization.

(5) There is little consumption before and after product demoulding, high demoulding times, low release agent and labor cost, and high production efficiency.

\section{Research status of semi-permanent release agent}

With the development of rubber products industry, the research of release agent has been carried out. However, the domestic research on semi-permanent release agent started late, and the domestic research on disposable release agent was mainly carried out. Domestic semipermanent release agent market has been monopolized by Daikin and other companies in Japan. With the excellent performance of semi-permanent release agent gradually emerges in recent years, domestic scholars begin to study[44].

Chem Trend developed a new generation of water based semi-permanent release agents that not only reduced the difficulty of release, but also provided a durable film. This semi-permanent, non-migrating coating was designed for all non-silicone, thermoplastic elastomers and polyaminoacetate rubber, especially for rubber processing[46].

Using perfluoropolyetheryl fluoride as raw material, Fan Yangyang[47] prepared perfluoropolyetheramide through hydrolysis and ammoniation. After emulsification, the water-based organic fluorine emulsion release agent with good chemical stability and heat stability was prepared. The product had long storage cycle and can realize semipermanent demoulding.

Zhang Chen[48] made organic fluorine emulsion release agent with perfluoropolymer and other raw materials, conducted infrared spectrum analysis and contact angle analysis, and discussed the influence of emulsifier type, dosage and other factors on the release agent. The prepared semi-permanent release agent can rapidly cure at $25^{\circ} \mathrm{C}$, and the release times can reach more than 20 times. Li Bin [41] developed two semi-permanent release agents that could be cured at room temperature based on organosilicon and organofluorine respectively. A crosslinking silicone resin was synthesized from two kinds of silanes and ethyl orthosilicate, and silicone oil and catalyst were added to prepare silicone release agent. The effects of resin type and amount of resin, amount of silicone oil and catalyst on the properties of release agent were studied, and then the curing properties were tested, and the effects of catalyst and release agent concentration on the curing properties were studied. Perfluoropolyetheryl was hydrolyzed and aminated into perfluoropolyetheramide, and then emulsified into organic fluorine release agent. The effects of emulsifier type and dosage, additives and other factors on the 
properties of the release agent were studied. The two semi-permanent release agents can be cured at $25^{\circ} \mathrm{C}$ with good stability and high release times.

Li Zhiming [49] et al. developed a semi-permanent release agent using polysiloxane and catalyst as raw materials, which had the advantages of low curing conditions, low cost and easy-to-use, and was suitable for epoxy resin products and rubber products.

Zhu Hang [50] et al. invented a water-based semipermanent release agent with a variety of fluorinecontaining compounds as raw materials. Compared with organosilicon release agent, the surface tension is lower and the release efficiency was higher, and the stripping times of the release agent can reach 23 times.

\section{Conclusion}

Semi-permanent release agent not only has the characteristics of high demoulding times, no pollution, low cost, less manual cleaning. It can also ensure the smooth surface of products and molds, and its excellent performance is unmatched by disposable release agent. However, high-end products have been dependent on imports, domestic research is relatively backward, causing a huge hindrance to the production of rubber, plastic and other industries. In order to break the monopoly situation of foreign companies, save costs, improve productivity and protect the environment, it is of great significance to develop semi-permanent release agents.

\section{Acknowledgments}

Thanks to the following funds for supporting this project. Heilongjiang Provincial Natural Science Foundation of China (LH2020E089), Postdoctoral Innovation Project of Shandong Province (202103051), China Postdoctoral Science Foundation (2021M691970), University Nursing Program for Young Scholars with Creative Talents in Heilongjiang Province (UNPYSCT-2018205), Fundamental Research Fundation for Universities of Heilongjiang Province (2018-KYYWF-1598).

\section{References}

[1] Zhigang Lei. Preparation and application of fluorine-containing demoulding agent[D]. Zhejiang University of Technology,2009.

[2] Amra, Kesrin, Momin, etc. Formulation evaluation of ketoconazole microemulsionloaded hydrogel with nigella oil as a penetration enhancer[J]. Journal of Cosmetic Dermatology, 2019, 18(6): 1742-1750.

[3] Benito J. M., Cambiella A., Lobo A., etc. Formulation, characterization and treatment of metalworking oil-in-water emulsions[J]. Clean Technologies and Environmental Policy, 2010, 12(1): 31-41.

[4] Beshkar Farshad, Khojasteh Hossein, SalavatiNiasari Masoud. Recyclable magnetic superhydrophobic straw soot sponge for highly efficient oil/water separation[J]. Journal of Colloid and Interface Science, 2017, 497(56): 57-65.

[5] Cambiella Angel, Benito Jose A., Pazos Carmen, etc. Interfacial properties of oil-in-water emulsions designed to be used as metalworking fluids $[J]$. Colloids and Surfaces aPhysicochemical and Engineering Aspects, 2007, 305(1-3): 112-119.

[6] Cambiella A., Benito J. M., Pazos C., etc. The effect of emulsifier concentration on the lubricating properties of oil-in-water emulsions[J]. Tribology Letters, 2006, 22(1): 5365.

[7] Cappelletti G., Ardizzone S., Meroni D., etc. Wettability of bare and fluorinated silanes: A combined approach based on surface free energy evaluations and dipole moment calculations[J]. Journal of Colloid and Interface Science, 2013, 389(557): 284-291.

[8] Carvalho De Almeida C., De Araujo Melo D. M., Maciel M. A. M., etc. Use of methanolic extract of ixora coccinea linn for corrosion inhibitor, which comprises preparation, characterization and application of microemulsion system based on extract as corrosion inhibitor, containing oil phase and aqueous phase. Maciel M a M: 英国.

[9] Cerpnjak Katja, Zvonar Alenka, Vrecer Franc, etc. Development of a solid selfmicroemulsifying drug delivery system (smedds) for solubility enhancement of naproxen[J]. Drug Development and Industrial Pharmacy, 2015, 41(9): 1548-1557.

[10] Chaydarreh Kamran Choupani, Shalbafan Ali, Welling Johannes. Effect of ingredient ratios of rigid polyurethane foam on foam core panels properties[J]. Journal of Applied Polymer Science, 2017, 134(17): 224-675.

[11] Chouhan P., Saini T. R. D-optimal design and development of microemulsion based transungual drug delivery formulation of ciclopirox olamine for treatment of onychomycosis[J]. Indian Journal of Pharmaceutical Sciences, 2016, 78(4): 498-511. deDamborenea J., Bastidas J. M., Vazquez A. J. Adsorption and inhibitive properties of four primary aliphatic amines on mild steel in $2 \mathrm{~m}$ hydrochloric acid[J]. Electrochimica Acta, 1997, 42(3): 455-459.

[13] Erdal M. Sedef, Ozhan Gul, Mat M. Cem, etc. Colloidal nanocarriers for the enhanced cutaneous delivery of naftifine: Characterization studies and in vitro and in vivo evaluations[J]. International Journal of Nanomedicine, 2016, 11(6): 1027-1037.

[14] Figueiredo Leandro, Bandeira Pedro, Mendes Adelio, etc. Use of fluoropolymer permanent release coatings for molded polyurethane foam production $[\mathrm{J}]$. Journal of Coatings Technology and Research, 2012, 9(6): 757-764. 
[15] Fu Yanhui, Li Zhijun, Liu Qinqin, etc. Construction of carbon nitride and mos2 quantum dot $2 \mathrm{~d} / 0 \mathrm{~d}$ hybrid photocatalyst: Direct z-scheme mechanism for improved photocatalytic activity[J]. Chinese Journal of Catalysis, 2017, 38(12): 2160-2170.

[16] Sirtl Erhard, . Über die reduktion von chlorsilanen mit wasserstoff[J]. Reuschel, 2004, 332(3-4): 113-123.

[17] Fu Yanhui, Liang Wei, Guo Jinqiu, etc. Mos2 quantum dots decorated g-c3n4/ag heterostructures for enhanced visible light photocatalytic activity[J]. Applied Surface Science, 2018, 430(3): 234-242.

[18] Kabakci Elif, Sayer Goksin, Suvaci Ender, etc. Processing-structure-property relationship in rigid polyurethane foams[J]. Journal of Applied Polymer Science, 2017, 134(21): 354-554.

[19] Kim S. H., Park H. C., Jeong H. M., etc. Glass fiber reinforced rigid polyurethane foams[J]. Journal of Materials Science, 2010, 45(10): 2675-2680.

[20] Kumar Neeraj Shishu. D-optimal experimental approach for designing topical microemulsion of itraconazole: Characterization and evaluation of antifungal efficacy against a standardized tinea pedis infection model in wistar rats[J]. European Journal of Pharmaceutical Sciences, 2015, 67(7): 97-112.

[21] Li Caifu, Mei Zhen, Liu Qian, etc. Formation and properties of paraffin wax submicron emulsions prepared by the emulsion inversion point method[J]. Colloids and Surfaces aPhysicochemical and Engineering Aspects, 2010, 356(1-3): 71-77.

[22] Li Guo, Dong Lei, Bai Zhu'an, etc. Predicting carbonation depth for concrete with organic film coatings combined with ageing effects[J]. Construction and Building Materials, 2017, 142(6): 59-65.

[23] Kim Kwang J., Shahinpoor Mohsen A novel method of manufacturing three-dimensional ionic polymer-metal composites (ipmcs) biomimetic sensors, actuators and artificial musclesj[J]. Polymer, 2015, 43(3): 797-802.

[24] Masuda Jun, Yan Jiwang, Zhou Tianfeng, etc. Thermally induced atomic diffusion at the interface between release agent coating and mould substrate in a glass moulding press[J]. Journal of Physics D-Applied Physics, 2011, 44(21): 345-677.

[25] Haowei Zhao. Preparation of self-emulsifying block silicone oil[J]. New Chemical Materials, 2019, 2(1): 1-4.

[26] Otsuka Yuma, Hiwasa Shin, Taniguchi Jun. Development of release agent-free replica mould material for ultraviolet nanoimprinting $[\mathrm{J}]$. Microelectronic Engineering, 2014, 123(53): 192-196.

[27] Peres Francois, Mofakhami Arthur Rapid tooling from protoypes: The cool-spray mold- making method[J]. Proceedings of SPIE - The International Society for Optical Engineering, 1998, 3517(11): 79-90.

[28] Scaffaro R., Maio A., Gulino F. E., etc. Bilayer biodegradable films prepared by co-extrusion film blowing: Mechanical performance, release kinetics of an antimicrobial agent and hydrolytic degradation[J]. Composites Part a-Applied Science and Manufacturing, 2020, 132(76): 453658.

[29] Serre C., Vayer M., Erre R. Behavior of internal mold release agent during bmc thermosets composites cure and aging $[\mathrm{J}]$. Journal of Materials Science Letters, 2001, 20(21): 19891991.

[30] Lotierzo A., Pifferi V., Ardizzone S., etc. Insight into the role of amines in metal working fluids [J] Corrosion Science, 2016, 110(3): 192-199.

[31] Migahed M. A., Abd-El-Raouf M., Al-Sabagh A. M., etc. Corrosion inhibition of carbon steel in acid chloride solution using ethoxylated fatty alkyl amine surfactants[J]. Journal of Applied Electrochemistry, 2006, 36(4): 395-402.

[32] Miyata Takahiro, Matsumoto Kozo, Endo Takeshi, etc. Synthesis and radical polymerization of styrene-based monomer having a five-membered cyclic carbonate structure[J]. Journal of Polymer Science Part aPolymer Chemistry, 2012, 50(15): 3046-3051.

[33] Negi Ripudaman Singh, Bisht Rajendra Singh, Singh Raj K. Physico-mechanical and antibacterial properties of pine gum/epoxy composites with/without silver nanoparticles[J]. Macromolecular Materials and Engineering, 2019, 304(7): 335-577.

[34] Neis Franciele Antonia, de Costa Fernanda, Fuller Thanise Nogueira, etc. Biomass yield of resin in adult pinus elliottii engelm. Trees is differentially regulated by environmental factors and biochemical effectors[J]. Industrial Crops and Products, 2018, 118(3): 20-25.

[35] Padula C., Telo I., Di Ianni A., etc. Microemulsion containing triamcinolone acetonide for buccal administration[J]. European Journal of Pharmaceutical Sciences, 2018, 115(2): 233-239.

[36] Pal Kaushik, Rajasekar R., Kang Dong Jin, etc. Effect of fillers on natural rubber/high styrene rubber blends with nano silica: Morphology and wear[J]. Materials \& Design, 2010, 31(2): 677686.

[37] Guanghui Yuan. Study on preparation process of organic silicon release agent[D]. Qingdao University of Science and Technology,2013.

[38] Mingming Xu. Synthesis and application of functional organosilicon compounds[D]. Tianjin Polytechnic University,2017.

[39] Chengyong Jiang. Silicone release agent and its application[J]. Plastics Industry, 2008, (02): 7475.

[40] Runlin Li. Development and characteristics of water - based semi-permanent release agent[J]. 
Rubber Reference, 1992, (05): 22-28.

[41] Bin Li. Study of room temperature curing semipermanent release agent[D]. Dalian Polytechnic University,2015.

[42] Hongsheng Guo. Development and characteristics of water - based semi-permanent release agent[J]. Rubber translations, 1993, (03): 19-24+3.

[43] Liying Wu, jianjun Gao, Wugang Jin. The release agent of composites metalized by transfer[J]. Colloids and Polymers, 2001, (04): 21-22.

[44] Bin Li, Hongyi Hai, Jianlou Ge, etc. Preparation and performance of semi-permanent release agent cured at room temperature[J]. Journal of Dalian Polytechnic University, 2015, 34(05): 345-348.

[45] Haibin Zhou. Use semi-permanent release agent to help release mold and prevent mold contamination[J]. World rubber Industry, 2007 , (01): 33-36.

[46] Xuezhong Tu. Semi-permanent release agent for precision model products[J]. Rubber Industry, 1996, (03): 184.

[47] Yangyang Fan, Bin Lin, Chen Zhang, etc. Preparation property of semi permanent mold release agent at room temperature[J]. New chemical materials, 2018, 46(03): 200-202+206.

[48] Chen Zhang, Bin Li, Yanjun Liu. Preparation of semi-permanent release agent cured at room temperature[J]. Technology Outlook, 2017, (4): 38.

[49] Zhiming Li, Wentao Xing, Jianyan $\mathrm{Xu}$, etc. Preparation method and application of a release agent. 2010.

[50] Hang Zhu, Xinliang Peng. A water-based semipermanent organic fluorine release agent. 2019. 